\title{
ENHANCEMENT OF DISSOLUTION AND THE ANTI- INFLAMMATORY EFFECT OF NIMESULIDE, USING LIQUISOLID COMPACT FOR ORAL APPLICATION
}

\author{
M. A. Hassan and H. M. El-Saghir
}

Department of Pharmaceutics, Faculty of Pharmacy, King Saud University

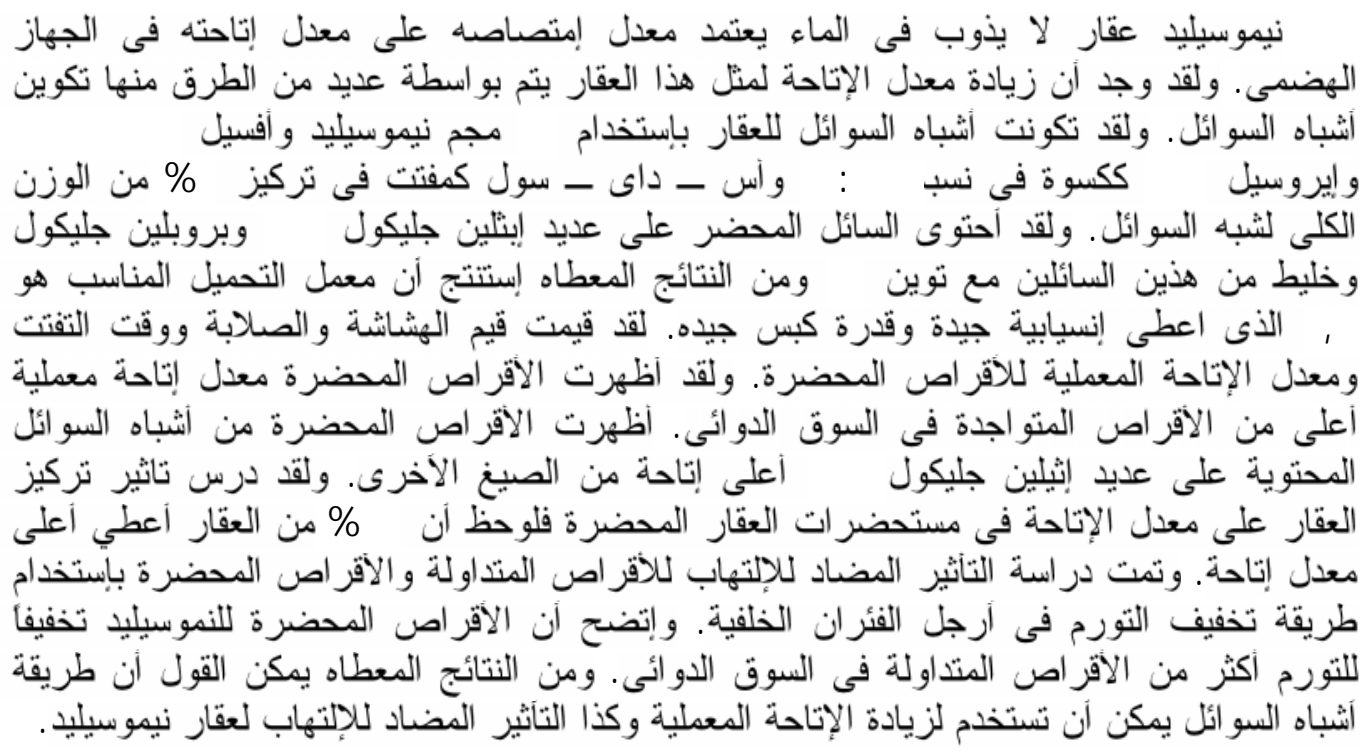

Nimesulide is a poorly soluble drug, the rate of its oral absorption is often controlled by the dissolution rate in the gastrointestinal tract. There are several techniques to enhance the dissolution of poorly soluble drugs. Among them the technique of liquisolid compacts which is a promising one. The liquisolid compacts were prepared using $20 \mathrm{mg}$ nimesulide, Avicel PH102 as a carrier, and Aerosil 200 as a coating material in a ratio of 20:1, as well as AC-DI-SOL as a disintegrant in a concentration of $5 \%$ from the total weight of the compact. The liquids used include PEG400, PG, and a mixture of these solvents with Tween 80. From the results obtained it is concluded that the suitable loading factor $\left(L_{f}\right)$ is 0.2 which gave good flowability and compressibility. Friability, hardness, disintegration time and the dissolution rate were carried out. All the liquisolid compacts showed higher dissolution rate than the conventional tablets. The liquisolid compacts containing the PEG400 showed the highest dissolution rate than the other preparations. The effect of different concentrations of drug on the dissolution rate was studied, and it was observed that $20 \%$ of drug gave the maximum dissolution rate, and no significant increase of the dissolution rate with increasing the drug concentration. Conventional tablets and liquisolid compacts containing PG and PEG400 were tested for their antiinflammatory effects using paw oedema test. liquisolid compacts exhibited a pronounced inhibition of swelling than that of conventional tablets. In conclusion liquisolid compact of nimesulide can be used as a technique to improve the dissolution rate and the antiinflammatory effect of nimesulide.

\section{INTRODUCTION}

It is well established that the active ingredient in a solid dosage form must undergo dissolution before it is available for absorption from the gastrointestinal tract. The poor dissolution characteristics of water-insoluble drugs are a major challenge for pharmaceutical scientists. The absorption rate of a poorly water-soluble drug, formulated as an orally administered solid dosage form, is controlled by its dissolution rate in the fluid present at the 
absorption site, i.e. the dissolution rate is often the rate-determining step in drug absorption ${ }^{1}$. There are several methods for enhancing dissolution rate of poorly water-soluble drugs including: (a) reducing particle size to increase surface area, thus increasing dissolution rate of drug; (b) solubilization in surfactant systems; (c) formation of water-soluble complexes; (d) drug derivatization such as a strong electrolyte salt forms that usually have higher dissolution rate and (e) manipulation of solid state of drug substance to improve drug dissolution, i.e. by decreasing crystallinity of drug substance through formation of solid solutions ${ }^{2}$. The most common method is to increase surface area of the drug by micronization. But, in practice the effect of micronization is often disappointing, especially when the drugs are encapsulated or tableted. Micronized drugs also have the tendency to agglomerate as a result of their hydrophobicity, thus reducing their available surface area. Several researchers have shown that the liquisolid technique is the most promising method for promoting dissolution rate of poorly water-soluble ${ }^{3-7}$.

From the historical point of view, liquisolid compacts were evolved from 'Powdered Solutions' which depends on preparing a true solution of the drug in a high boiling point, water-miscible solvent, which was carried out on the extensive surface of an inert carrier such as silica ${ }^{8}$.

In such systems, the drug existed in a molecular state of subdivision'. Also, these systems were free flowing, non-adherent, dry looking powders ${ }^{10}$. In further studies, compression enhancers were added to these powdered solutions, as microcrystalline cellulose. However, the compression of these systems may result in 'Liquid Squeezing Out' phenomenon ${ }^{6}$. Liquisolid compacts (possess acceptable flowability and compressibility properties), are prepared by simple blending with selected powder excipients referred to as the carriers and the coating materials. Many grades of cellulose, starch, lactose, etc. can be used as carriers, whereas silica of very fine particle size can be used as coating materials. The liquid medication is to be mixed with the excipients and then compressed into tablets. It was proved that the smaller the drug concentration in the liquid medication, the more rapid the release rates, since drugs in a high concentration tend to precipitate within the silica pores ${ }^{11}$.

Nimesulide, a non-steroidal antiinflammatory drug (NSAID), is administered orally or rectally twice daily for a variety of inflammation and pain states. This is a unique NSAID, not only because of its chemical structure but also because of its specific affinity to inhibit cyclooxygenase-2 (COX-2), thus exerting milder effects on the gastrointestinal mucosa ${ }^{12}$.

The aim of this study was to increase dissolution rate of nimesulide using liquisolid technique. It was formulated into $20 \mathrm{mg}$ drug in liquisolid tablets consisting of similar powder excipients and different non-volatile solvents as polyethylene glycol 400 (PEG400), propylene glycol (PG), and mixtures of these solvents as well as Tween $80(1: 1)$ in their liquid medications. The loading factor and the flowability of the powder were determined. The prepared liquisolid compact was evaluated according to hardness, friability and disintegration time. The in-vitro drug dissolution rates of formulated liquisolid compacts were compared to those of conventionally prepared (directly compressed) tablets using a USP dissolution apparatus at dissolution media ( $\mathrm{pH}$ 1.2). The antiinflammatory effect of liquisolid compacts was also tested using paw oedema test.

\section{MATERIALS AND METHODS}

\section{Materials}

Nimesulide was purchased by Triveni chemicals, Vapi (India), Avicel PH102 (seppic, France) Aerosil 200 (FMC co. Philadelphia, USA), Ac-Di-SOL (Cooperation Pharmaceutique Francaise, France), PEG400, PG and Tween 80 (Merck, Germany), all other chemicals were of analytical grades.

\section{Methods \\ Determination of the loading factor}

PEG400, PG, and mixture of both solvents as well as Tween 80 in a ratio of 1:1 were used as liquids in the preparation of liquisolid compacts. It has been established that, for a given powder substrate consisting of a certain carrier and a coating powder mixed at various ratios $(R)$, there are specific maximum liquid loading factor $\left(L_{f}\right)$ which must be employed in 
order to produce acceptably flowing liquisolid systems. The relation between these factors is presented by the following equation:

$$
R=Q / q
$$

Where $(Q)$ is the carrier and $(q)$ is the coating material.

Such a characteristic amount of liquid is termed the liquid load factor $\left(L_{\mathrm{f}}\right)$ and defined as the weight ratio of the liquid medication $(W)$ and carrier powder $(Q)$ in the system, i.e.:

$$
L_{\mathrm{f}}=W / Q
$$

\section{Determination of flow properties}

The flow properties were assessed through measuring the compressibility index $\mathrm{Ci} \%^{13}$. An amount of the prepared powder of $20 \mathrm{~g}$ was accurately weighed, passed through a $1 \mathrm{~mm}$ screen to break up agglomerates and placed in a $50 \mathrm{ml}$ volumetric cylinder without compaction. The apparent volume $V o$ was recorded. Then the cylinder was tapped by raising it to a height of $12-14 \mathrm{~mm}$ and then allowing it to fall under its own weight. This was repeated until no change in volume occurred. This final volume $V f$ was recorded.

The $\mathrm{Ci} \%$ was determined from the following relation:

$$
\mathrm{Ci} \%=(\mathrm{Vo}-\mathrm{Vf}) / \mathrm{Vo} \times 100
$$

The smaller the value of the $C i \%$, the better will be the flow properties of the powder ${ }^{14}$.

Flow properties of the powders were also evaluated by determining the angle of repose. Static angle of repose was measured according to the fixed funnel and freestanding cone method. A funnel with the end of the stem cut perpendicular to the axis of symmetry is secured with its tip $10 \mathrm{~cm}$ height, $H$, above a graph paper placed on a flat horizontal surface. The powders were carefully poured through the funnel until the apex of the conical pile so formed just reaches the tip of the funnel. The mean diameter, $2 R$, of $H$, base of the powder cone, was determined and the tangent of the angle of repose was given by:

$$
\tan \alpha=H / R
$$

Where $\alpha$ is the repose angle.

Table 1 represents the different loading factors ranging from 0.1-0.4 to determine the optimum loading factor which gives good flowability and compressibility.

Table 1: Different formulae using different loading factor.

\begin{tabular}{|c|c|c|c|c|}
\hline $\begin{array}{c}\text { Formula } \\
\text { No }\end{array}$ & $\begin{array}{c}\text { Loading } \\
\text { factor } \\
\mathrm{L}_{\mathrm{f}}\end{array}$ & $\begin{array}{c}\text { Weight } \\
\text { of carrier } \\
(\mathrm{mg})\end{array}$ & $\begin{array}{c}\text { Weight of } \\
\text { adsorbent } \\
(\mathrm{mg})\end{array}$ & $\begin{array}{c}\text { Weight } \\
\text { of liquid } \\
(\mathrm{mg})\end{array}$ \\
\hline F1 & 0.15 & 500 & 25 & 75 \\
F2 & 0.2 & 500 & 25 & 100 \\
F3 & 0.25 & 500 & 25 & 125 \\
F4 & 0.3 & 500 & 25 & 150 \\
F5 & 0.35 & 500 & 25 & 175 \\
F6 & 0.4 & 500 & 25 & 200 \\
\hline
\end{tabular}

\section{Preparation of conventional tablets and liquisolid compacts}

Nimesulide conventional tablets were prepared by mixing the drug $(20 \mathrm{mg}$ for each tablet) with microcrystalline cellulose-Aerosil 200 in a ratio of 20:1 for a period of $10 \mathrm{~min}$ in a cubic mixer (Erweka, TypeUG, Germany). The mixture was mixed with Ac-Di-Sol (5\%, $\mathrm{w} / \mathrm{w}$, of the formulation) for $10 \mathrm{~min}$, and then it was compressed on a $10-\mathrm{mm}$ punch and die using a manual tablet machine (Riken, Japan). Sufficient compression loads were applied in order to produce tablets with the hardness of 6$7 \mathrm{~kg}$. Several liquisolid compacts, denoted as F1 to F-8 were prepared as follows: nimesulide was dispersed in PEG400, PG, and mixture of PEG400 or PEG as well as Tween 80 in a ratio $1: 1$.Then a binary mixture of carrier-coating materials (microcrystalline cellulose as the carrier powder and Aerosil 200 as the coating material) was added to the obtained liquid medication under continuous mixing in a mortar. Finally, 5\% (w/w) of Ac-Di-Sol as the disintegrant, was mixed with the mixture for a period of $10 \mathrm{~min}$. Then the final mixture was compressed. Important formulation characteristics of the prepared nimesulide liquisolid formulations are shown in table 2 .

\section{Evaluation of nimesulide liquisolid tablets \\ 1. Differential Scanning Calorimetery (DSC) of the prepared liquisolid powder \\ Prior to the compression of the} formulations into tablets, pre-compression studies were carried out using differential scanning calorimetry (DSC) in order to ensure the suitability of the selected excipients. 
Table 2: Formulation characteristics of the prepared nimesulide liquisolid compacts.

\begin{tabular}{|c|c|c|c|c|c|c||}
\hline $\begin{array}{c}\text { Formula } \\
\text { No }\end{array}$ & $\begin{array}{c}\text { Drug } \\
\%\end{array}$ & $\begin{array}{c}\text { Amount } \\
\text { of liquid } \\
(\mathrm{mg})\end{array}$ & $\begin{array}{c}\text { Amount of } \\
\text { carrier }(\mathrm{mg}) \\
\mathrm{Q}\end{array}$ & $\begin{array}{c}\text { Amount of } \\
\text { coating (m) } \\
\mathrm{q}\end{array}$ & $\begin{array}{c}\text { Amount of } \\
\text { disintegran } \\
\mathrm{t} \\
(\mathrm{mg})\end{array}$ & $\begin{array}{c}\text { Total } \\
\text { weight } \\
\text { of tablet }\end{array}$ \\
\hline F1 & 10 & 200.00 & 1100 & 55.00 & 68.75 & 1443.80 \\
F2 & 20 & 100.00 & 600 & 30.00 & 37.50 & 787.50 \\
F3 & 30 & 66.6 .00 & 435 & 21.75 & 27.20 & 669.20 \\
F4 & 40 & 50.00 & 350 & 17.50 & 21.88 & 459.40 \\
F5 & 50 & 40.00 & 300 & 15.00 & 18.75 & 393.80 \\
F6 & 20 & 100.00 & 600 & 30.00 & 37.50 & 787.50 \\
F7 & 20 & 100.00 & 600 & 30.00 & 37.50 & 787.50 \\
F8 & 20 & 100.00 & 600 & 30.00 & 37.50 & 787.50 \\
\hline
\end{tabular}

Amount of drug $=20 \mathrm{mg}$; Loading factor $\left(\mathrm{L}_{\mathrm{f}}\right)=0.2$; Ratio of carrier $:$ coat $(\mathrm{R})=20$.

Differential scanning calorimetry (DSC) was performed using Shimadzu differential scanning calorimeter (DSC-50, Kyoto, Japan), the thermal behaviors of the drug (nimesulide), Avicel PH 102, Aerosil 200, as well as the liquisolid system prepared was determined. Samples of 3-4 mg of the pure nimesulide or the above-mentioned samples were sealed in aluminum pans at a constant heating rate of $5^{\circ} \mathrm{C} / \mathrm{min}$ in the scanning temperature range of 0 to $200^{\circ} \mathrm{C}$. Empty aluminum pans were used as references and the whole thermal behaviors were studied under a nitrogen purge.

\section{Determination of the drug content}

The prepared nimesulide liquisolid tablets of the selected formulae were evaluated. Nimesulide content in different liquisolid tablet formulations was determined by accurately weighing 10 tablets of each formula individually. Each tablet was then crushed and dissolved in $100 \mathrm{ml} 0.1 \mathrm{~N} \mathrm{HCl}$, then, the solution was filtered, properly diluted, and then measured spectrophotometrically using Spectrophotometer (UV-1601 PC Shimadzu, Japan) at $\lambda_{\max } 296 \mathrm{~nm}$, thereafter, the nimesulide content of each tablet was determined. The drug content was found to be between $96.5-98.8 \%$ for all the formulae prepared.

\section{Determination of the friability, hardness, and disintegration time}

The friability of the prepared formulae was measured using Digital tablet friability tester, (ERWEKA- Germany), and the percentage loss in weights were calculated and taken as a measure of friability. The hardness of the liquisolid tablets prepared was evaluated using Stoke's hardness tester (ERWEKAGermany), the mean hardness of each formula was determined. The disintegration time was performed using USP disintegration tester, VTD-3 (Progressive Incorp., Bombay, India). The friability was found not more than $1 \%$, and the hardness between $6-7 \mathrm{Kg}$ of all formulae, and the disintegration time about 15 minutes for liquisolid compacts, and the compressed tablets.

\section{Dissolution study}

Finally, the in-vitro dissolution studies were carried out and the dissolution rate of nimesulide from liquisolid tablets were determined using the USP paddle dissolution method (Erweka, DPT6R, Germany). In this method, $0.1 \mathrm{~N}$ hydrochloric acid ( $\mathrm{pH} 1.2)$ was used as a dissolution medium. The rate of stirring was $50 \pm 2 \mathrm{rpm}$. One tablet was placed in $900 \mathrm{ml}$ of dissolution medium and maintained at $37 \pm 0.1^{\circ} \mathrm{C}$. At appropriate time intervals $(5,10,15,20,30,45$ and $60 \mathrm{~min}), 5$ $\mathrm{ml}$ of the samples were taken and filtered through a 0.45 um Millipore filter. The dissolution medium was then replaced by $5 \mathrm{ml}$ of fresh dissolution fluid to maintain a constant volume. The samples were then analyzed at $296 \mathrm{~nm}$ spectrophotometetrically. The mean of six determinations was used to calculate the drug dissolved from each of the prepared formulations. 


\section{Determination of anti-inflammatory effect of nimesulide using liquisolid compact}

Male rats (4 per group) weighing $200 \pm 20$ $\mathrm{g}$ were used. The rats were fasted with free access to water for $12 \mathrm{hrs}$ prior to the test. Oedema was induced by subcutaneous injection of $1 \%$ carrageenan into the right subplanter region. The thickness of the rat hind paw was measured by Vernier Caliper (SMEC, China) before injection of carageenan, immediately after injection, and every hour for 4 hrs. The liquisolid compact containing nimesulide and the direct compression tablets were administered perorally, $1 \mathrm{hr}$ after carageenan injection. The control group received the same formula without the drug. The percent swelling of the paw was calculated using the following equation:

$$
\% \text { swelling }=[(\mathrm{V}-\mathrm{Vi}) / \mathrm{Vi}] \times 100
$$

Where $V$ is the paw thickness at each time interval, and $V i$ is the initial paw thickness before carrageenan injection. The average paw swelling in treated rats was compared with that of control rats and the percent inhibition of oedema was determined using the following equation:

\section{$\%$ inhibition $=[1-(\%$ swelling of treated group $1 \%$ swelling of control group) $] \times 100$}

The percent inhibition of swelling was plotted against time to compare the antiinflammatory effect of the different formulae.

\section{RESULTS AND DISCUSSION}

The very poor aqueous solubility and wettability of cox-2 inhibitors however gives rise to difficulties in the design of pharmaceutical formulations and leads to a variable oral bioavailability. Solubility of nimesulide was determined in different solvents as propylene glycol, polyethylene glycol 400, glycerol and water. The solubility of the drug in different solvents was 63.120, $1.760,0.218$, and $0.014 \mathrm{mg} / \mathrm{ml}$ for PEG400, PG, glycerol and water respectively ${ }^{15}$.

\section{Determination of loading factor $\left(L_{f}\right)$}

Different loading factors $\left(L_{f}^{\prime} s\right)$ were used ranging from 0.1 to 0.4 to obtain the optimum $L_{f}$ which gives the highest flowability and compressibility. Table 2 represents different $L_{f}$ used. From the results obtained it was observed that $0.2 L_{f}$ was optimum loading factor, which give good flowability and compressibility, and it is close to those recommended in the literature $^{5 \& 6}$. The $L_{f} 0.2$ is the commonly used loading factor.

\section{Determination of powder Flowability}

As the angle of repose $(\alpha)$ is characteristic of the internal friction or cohesion of the particles, the value of the angle of repose will be high if the powder is cohesive and low if the powder is non-cohesive. As presented in table $3, F 1$ and F2 showed $(\alpha)$ values of 33.50 and 35.00 respectively were chosen as liquisolid systems with acceptable flowability, while those having higher angles of repose were considered as non-acceptable. Powders showing Carr's index (Ci) up to 21 are considered of acceptable flow properties ${ }^{16}$.

F1 and F2 possessed the best compressibility due to having the least $\mathrm{Ci} \%$, and upon compression, resulted in tablets of uniform weight, and acceptable (Table 3). Thus the rest of investigation was concentrated on using 0.2 as a liquid loading factor.

Table 3: Determination of angle of repose and Carr's index of the prepared powders.

\begin{tabular}{|c|c|c|c||}
\hline $\begin{array}{c}\text { Formula } \\
\text { No }\end{array}$ & $\begin{array}{c}\text { Loading } \\
\text { factor } \\
\mathrm{L}_{\mathrm{f}}\end{array}$ & $\begin{array}{c}\text { Average angle } \\
\text { of repose } \\
\alpha \pm \mathrm{SD}\end{array}$ & $\begin{array}{c}\text { Average } \\
\text { Carr's index } \\
(\mathrm{Ci}) \% \pm \mathrm{SD}\end{array}$ \\
\hline F1 & 0.15 & $33.50 \pm 1.20$ & $19.96 \pm 0.96$ \\
F2 & 0.20 & $35.00 \pm 0.90$ & $20.23 \pm 1.02$ \\
F3 & 0.25 & $38.00 \pm 1.12$ & $21.86 \pm 0.82$ \\
F4 & 0.30 & $39.75 \pm 0.75$ & $23.58 \pm 0.64$ \\
F5 & 0.35 & $44.50 \pm 0.96$ & $26.62 \pm 0.34$ \\
F6 & 0.40 & $46.83 \pm 1.40$ & $29.44 \pm 0.55$ \\
\hline
\end{tabular}

\section{DSC study}

One of the most classic applications of DSC analysis is the determination of the possible interactions between a drug entity and the excipients in its formulation; it is very important to establish the existence of any incompatibilities during the preformulation stage to ensure the success of the subsequent stability studies. Figure 1 reveals the thermal behaviors of the pure components together with the thermal behavior of the final liquisolid system prepared. Nimesulide peak is clear in its 
DSC thermogram (Fig. 1A) demonstrating a sharp characteristic endothermic peak at $149.25^{\circ} \mathrm{C}$ corresponding to its melting temperature $(\mathrm{Tm})$; such a sharp endothermic peak signifies that nimesulide used was in pure crystalline state. The thermograms of Avicel PH 102 (Fig. 1B) showed no endothermic peak. The thermal behavior of Aerosil 200 in figure $1 \mathrm{C}$ did not show any sharp peaks; proving that the carrier and coating material were almost in an amorphous state. The liquisolid system thermogram in figure 1 displayed complete disappearance of characteristic peak of nimesulide a fact that agrees with the formation of drug solution in the liquisolid powdered system, i.e., the drug was molecularly dispersed within the liquisolid matrix. Such disappearance of the drug peaks upon formulation of the liquisolid system declared that the complete suppression of all drug thermal features, undoubtedly indicates the formation of an amorphous solid solution. Additionally, the total disappearance of the drug melting peak indicates that drug amorphization was obtained $^{17}$.

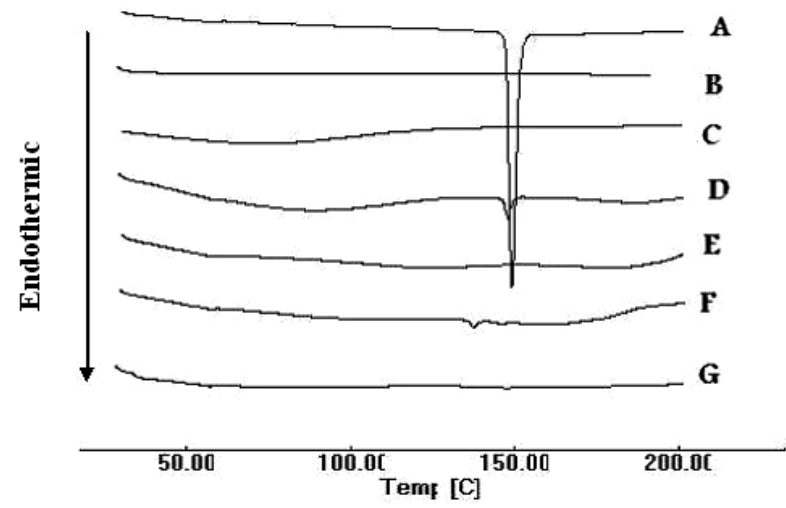

Fig. 1: DSC thermogram of nimesulide (A), Avicel PH102 (B), Aerosil 200 (C), physical mixture (D), liquisolid compact mixture containing PEG400 (E), liquisolid compact mixture containing PG (F), and liquisolid compact mixture containing Tween $80(\mathrm{G})$.

\section{Dissolution study of nimesulide liquisolid compacts}

The dissolution profiles of nimesulide from the liquisolid compacts (F1-F8) and conventional formulations (tablets) are shown in figure 2. In-vitro dissolution profiles of nimesulide showed that the liquisolid compacts produced higher dissolution rates in comparison with the conventional tablet. For example, the percentages of drug released from F1, F6, F7, F8, and conventional tablets after 1 hr were $100 \%, 80 \%, 100 \%, 88 \%$, and $50 \%$ at pH 1.2 respectively. This shows that the tablets containing PEG400, PG, and mixture of both solvent with Tween 80 in 1:1 ratio (liquisolid compacts) produced faster dissolution rate in comparison with compressed tablets. Such enhanced drug dissolution rates may be mainly attributed to the fact that this poorly watersoluble drug is already in solution in different solvents used (PEG400, PG, and both solvents with Tween 80), while at the same time, it is carried by the powder particles (microcrystalline cellulose-Aerosil) of the liquisolid vehicle. Thus, its release is accelerated due to its markedly increased wettability and surface availability to the dissolution medium. Figure 3 shows the drug dissolution rate $(D R)$ from different liquisolid compacts, and directly compressed tablets in the first $10 \mathrm{~min}$. As it is clear from this figure, that the liquisolid tablets displayed higher dissolution rates than those of directly compressed tablets (DCT).

$D R$ was calculated as follows:

$$
D R=(M \times D) / 1000
$$

Where $M$ is the total amount of nimesulide in each tablet (in this study, it is $20,000 \mathrm{~g}$ ) and $D$ denotes percentage of drug dissolved in the first $10 \mathrm{~min}$.

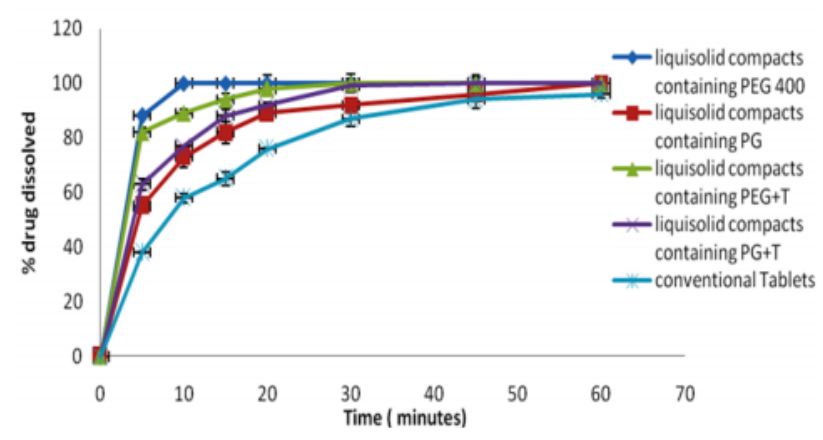

Fig. 2: The dissolution profile of nimesulide from different liquisolid compacts compared with conventional tablets.

Figure 3 shows the dissolution rates of different liquisolid compacts using different solvents (PEG400, PG, PEG+Tween, $\mathrm{PG}+\mathrm{Tween})$ in comparison with conventional tablets. The figure shows that dissolution rates 
decreased in the following order: PEG400 > PEG400+Tween > PG+Tween > PG > compressed tablets.

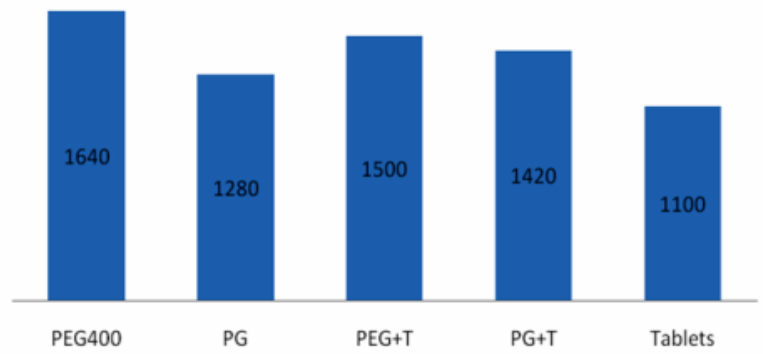

Fig. 3: The dissolution rates $(D R)$ of nimesulide from different liquisolid compacts and conventional tablets.

According to the classic dissolution equation (3):

$$
D R=(D / h) S(C s-C)
$$

The dissolution rate $(D R)$ of a drug is directly proportional to its concentration gradient $(C s-C)$ in the stagnant diffusion layer and its surface $(S)$ available for dissolution. $C S$ is the saturation solubility of the drug in the dissolution medium and, thus, it is a constant characteristic property related to the drug and dissolving liquid involved. Since all of dissolution tests for formulations were done at a constant rotational paddle speed $(50 \mathrm{rpm})$ and identical dissolving media, we can assume that the thickness $(h)$ of the stagnant diffusion layer and the diffusion coefficient $(D)$ of the drug molecules remain almost identical. Therefore, the observed higher dissolution rates of nimesulide from liquisolid tablets are due to the significantly increased surface of the molecularly dispersed nimesulide ${ }^{3}$. In addition, the saturation solubility of the drug in the microenvironment $(C s)$ might be increased in the liquisolid compacts.

The drug concentration in the liquid medication is one of the main factors on the performance of a liquisolid compact. The effect of drug concentration $(C d)$ in the liquid medication (PEG400) on dissolution profile of nimesulide from the liquisolid compacts in 0.1 $\mathrm{N} \mathrm{HCl}$ dissolution media is shown in figure 4. The figure shows that the increase of drug concentration from $20-50 \%$ in the liquid medication a liquisolid compact has no considerable effect on the dissolution rate. It can be seen that dissolution rate has no significant increase with an increase in the concentration of drug. This result can be attributed to that the drug in low concentrations is in a molecular form which is more soluble than the drug in higher concentrations ${ }^{3}$.

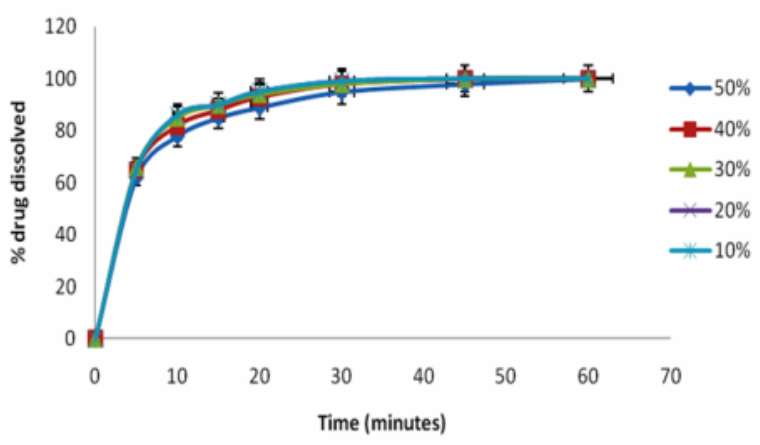

Fig. 4: Effect of different concentrations of drug on the dissolution from liquisolid compacts containing PEG.

\section{The anti-inflammatory effect of nimesulide from liquisolid compact}

The anti-inflammatory effect of nimesulide from two formulae of liquisolid compacts compared with conventional tablets. The percent inhibition observed with any formulae considered the maximum possible response for anti-inflammatory effect of nimesulide in this formula during the $4 \mathrm{hrs}$ observation period. Figure 5 showed the percent inhibition of paw edema plotted against time. From the figure it was observed that the order of suppression of swelling (percentage inhibition) was as follows: LSC (PEG) > LSC $(\mathrm{PG})>$ conventional tablets.

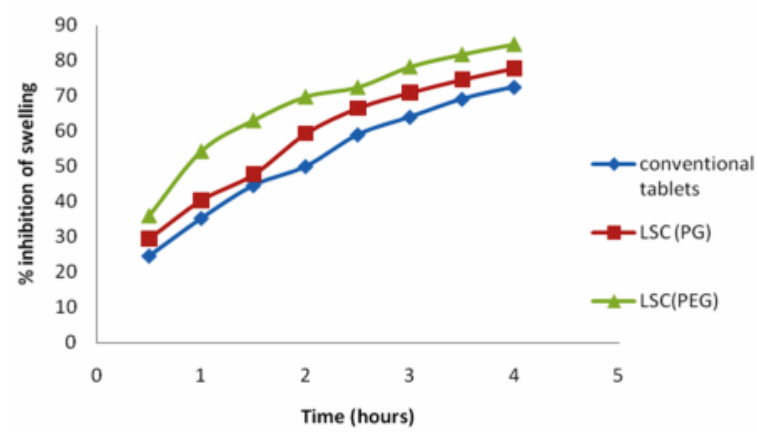

Fig. 5: The percentage inhibition of swelling from nimesulide liquisolid compacts compared with conventional tablets. 


\section{Conclusion}

The liquisolid technique is the most promising method for promoting dissolution rate of poorly water-soluble drugs. The results showed that the optimum loading factor which gives good flowability and compressibility is 0.2 , and the liquisolid compact containing PEG400 gives the highest dissolution rate than other formulae. Also the results showed no increase in the amount of the drug dissolved as the concentration of the drug increases. Liquisolid compacts give a pronounced antiinflammatory effect when compared with conventional tablets.

\section{Acknowledgment}

The authors are greatly thankful to research center, King Saud University, Female Section, Riyadh, KSA, for financial support in form of research fellowship.

\section{REFERENCES}

1- Y. Javadzadeh, J. N. Baharak and A. Nokhodchi, "Liquisolid technique for dissolution rate enhancement of a high dose water-insoluble drug (carbamazepine)", Int. J. Pharm., 341, 2634 (2007).

2- S. G. Kapsi and J. W. Ayres, "Processing factors in development of solid solution formulation of itraconazole for enhancement of drug dissolution and bioavailability", ibid., 229, 193-203 (2001).

3- Y. Javadzadeh, M. R. Siahi, M. BarzegarJalali and A. Nokhodchi, "Enhancement of dissolution rate of piroxicam using liquisolid compacts", IL Farmaco, 60, 361-365 (2005).

4- A. Nokhodchi, Y. Javadzadeh, M. R. Siahi and M. Barzegar-Jalali, "The effect of type and concentration of vehicles on the dissolution rate of a poorly soluble drug (indomethacin) from liquisolid compacts", J. Pharm. Pharmaceut. Sci., 8, 18-25 (2005).

5- S. Spireas and S. Sadu, "Enhancement of prednisolone dissolution properties using liquisolid compacts", Int. J. Pharm., 166, 177-188 (1998).

6- S. Spireas, S. Sadu and R. Grover, "Invitro release evaluation of hydrocortisone liquisolid tablets", J. Pharm. Sci., 87, 867872 (1998).

7- S. Spireas, T. Wang and R. Grover, "Effect of powder substrate on the dissolution properties of methchrothiazide liquisolid compacts", Drug Dev. Ind. Pharm., 25, 163-168 (1999).

8- A. Sheth and C. I. Jarowski, "Use of powdered solutions to improve the dissolution rate of polythiazide", ibid., 16, 769-777 (1990).

9- K. Y. Yang, R. Glenza and C. I. Jarowski, "Effects of amorphous silicon dioxides on drug dissolution", J. Pharm. Sci., 68, 560565 (1979).

10- C. C. Liao and C. I. Jarowski, "Dissolution rates of corticoid solutions dispersed on silicas", ibid., 73, 401-403 (1984).

11- K. A. Khaled, "Formulation and evaluation of hydrochlorothiazide liquisolid tablets", Saudi Pharm. J., 6, 3946 (1988).

12- K. Singlaa, M. Chawla and A. Singh, "Nimesulide: Some pharmaceutical and pharmacological aspects: An update", J. Pharm. Pharmacol., 52 (5), 467486 (2000).

13- H. G. Brittain, "Physical Characterization of Pharmaceutical Solids", Marcel Dekker Inc., New York, 1995, pp.294-297.

14- S. A. Tayel, I. I. Soliman and D. Louis, "Improvement of dissolution properties of Carbamazepine through application of the liquisolid tablet technique", Eur. J. Pharm. Biopharm., 69, 342-347 (2008).

15- N. Seedher and S. Bhatia, "Solubility enhancement of Cox-2 inhibitors using various solvent systems", AAPS PharmSciTech., 4 (3), 1-9 (2003).

16- J. Staniforth, "Powder Flow", In: M. Aulton (Ed.), "Pharmaceutics, the Science of Dosage Form Design", $2^{\text {nd }}$ ed., Churchill Livingstone, Longman group, Edinburgh, 2002, pp. 197-210.

17- R. H. Fahmy and M. A. Kassem, "Enhancement of famotidine dissolution rate through liquisolid tablets formulation: In-vitro and in-vivo evaluation", Eur. J. Pharm. and Biopharm., 69, 993-1003 (2008). 